\title{
Article
}

\section{Box-Behnken design of quadratic model for Gas Metal Arc Welding (GMAW) and optimization analysis on desirability function}

\author{
Kashif Nazir $^{1, *}$, Anwar Khalil Sheikh ${ }^{1}$ and Muhammad Asad Ahmad ${ }^{1}$ \\ 1 Department of Mechanical Engineering, King Fahd University of Petroleum and Minerals (KFUPM), Dhahran, \\ Kingdom of Saudi Arabia.; anwarks@kfupm.edu.sa(A. K); asadahmad13@gmail.com(M. A) \\ * Correspondence: kashif9003@gmail.com; Tel.: +97339253640
}

Received: 16 January 2019; Accepted: 25 March 2019; Published: 31 March 2019.

\begin{abstract}
Gas Metal Arc Welding (GMAW) is one of those welding processes which is increasingly being used in many varieties of fabrication and manufacturing industries due to high production rate and ease of work such as fewer fumes/smoke and less time chipping slag. Important parameters which affect GMAW process are amperage, arc length, wire feed speed, welding speed and welding voltage. In this paper, we have focused and studied important controllable factor's effects on depth of penetration, deposition efficiency, bead width and bead reinforcement to find best weld during welding in mobile or outdoor welding shop in stress and overloaded conditions by Box-Behnken design of quadratic model for GMAW process and optimization analysis on desirability function. Quality of welds is defined as "the level of perfection that welds exhibit pertaining to the entire volume of weldment as well as to the profile of weld surface appearance". With reference to quality weld definition as defined above, the study is performed to find out the best welding condition whereas best weld is the weld with deposition efficiency value as larger the best, depth of penetration value with "larger the best", bead width value "nominal the best", discontinuities number per weld's count value "smaller the best". Productivity in term of quality is defined as an optimum blend of parameters which inevitably develop minimum or no defect then the process will result in high productivity. For given certain material and similar welding circumstances, our analysis has found the most optimal factor's values for the similar condition of field data. This study will contribute welding research work in terms of points as described, firstly to enhance the knowledge of welding process and analysis by utilizing DOE along with desirability function, secondly ability to provide narrow window of weld process parameter to produce the quality weld and thirdly to study the GMAW process for mobile welding shop in the toughest condition such as the windy and dusty environment.
\end{abstract}

Keywords: Gas metal arc welding, amperage, arc length, travel speed, RSM, optimization.

\section{Introduction}

I n Gas Metal Arc Welding (GMAW), materials are melted and joined by heating them by an arc which is produced between filler wire which is continuously fed the metals. Arc is protected and shielded by using inert gases like Helium and Argon and because of this GMAW is also called Metal Inert Gas (MIG) process. Other than inert gases some active gases i.e. $\mathrm{CO}_{2}$ are used so GMAW is now the widely used name for this process. This is constant voltage process and Direct Current Electrode Positive (DCEN) is used in GMAW. Either Direct Current Electrode Positive (DCEN) or Alternating Current (AC) transfer of metal is unreliable. But here arc is a stable transfer of metal smoothly, low chances of spatters, and high penetration is worthy characteristics of this process [1,2].

M. Azadi [3] studied the optimization of GMAW over API-X42 material through L36 Taguchi matrix and proposed back propagation neural network (BPNN). Omer Bataineh [4] studied the GMAW process to find the effects of welding factors over welding strength; the optimization was done through factorial design methodology and ANOVA. Izzatul [5] optimized the welding responses such as penetration, microstructural and hardness by utilizing robotic GMAW process. Pawan Kumar [6] studied the dissimilar welding joints 
welded by GMAW and optimized the result by Taguchi orthogonal L9 array and analysis was concluded based on the signal to noise ratios. Chandresh [7] worked with full factorial design experiment to optimize the GMAW responses on AISI 1020 material.

All above investigation and experimental study were conducted on GMAW process under a controlled environment such as well-established and equipped closed welding workshop whereas we have studied the semi-automatic GMAW performed under tough environmental conditions such as windy and dusty in the mobile or outdoor welding shop. Through data mining of field engineering on nominal identical data on which we have done field study and relevant data points are chosen. The portable welding shop is the shop which moves the location to location base on projects scope such as for cross-country pipeline.

\section{Process Features and Parameters [1]}

In the gas metal welding, arc regulation is auto-adjusted by any of two ways. The most known technique is to employ a constant-speed electrode feed unit with a variable current and constant voltage source. With the variation of gap between gun and work piece, the variation in arc length is also noted, where if arc length decrease power source gives more current and if arc length increases then of course less current. So there will be relevant changes in meting rate due to such changes in current which in turn require maintaining arc length. Other technique of arc regulation is to employ a constant-current source along with adjustable-speed, voltage-sensing electrode feeder. Here any change in arc length originates a relevant variation in the AC voltage. Based on the electrode feed unit changes the speed of filler metal wire depending upon higher/lower speed. This is more useful for larger electrodes when lower feed speed of filler metal wire is required. The GMAW setup used for the research work is depicted in Figure 1.
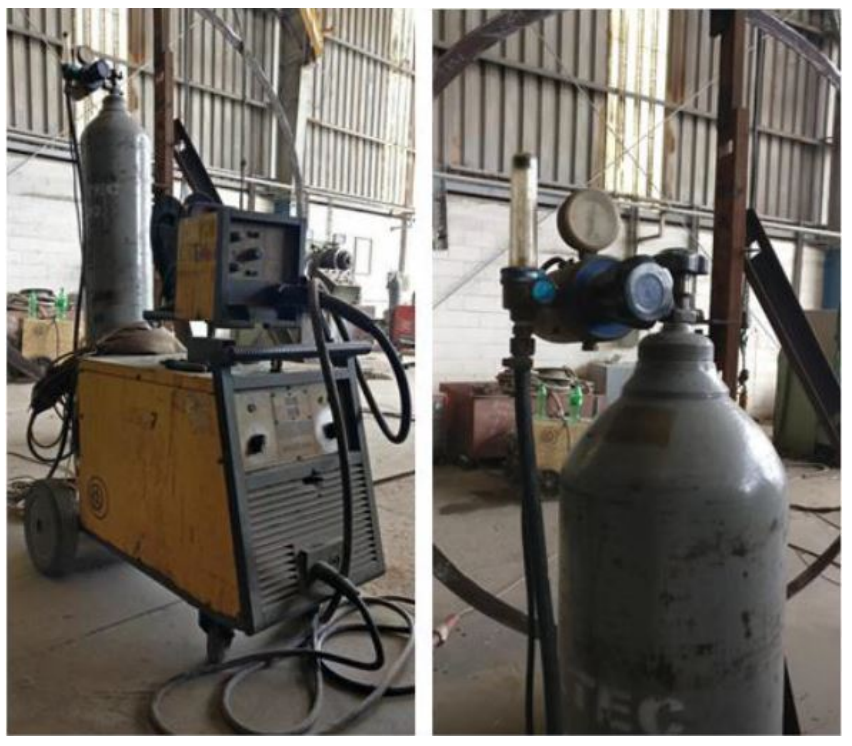

Figure 1. Gas metal arc welding (GMAW) process setup used for welding data collection

\section{Design of Experiment Methodology [8,9]}

The factors can be classified as either continuous with low and high value or categorical with different level. We have selected continuous type of factors instead of the categorical type with minimum and maximum value because GMAW factor's values fluctuate frequently due to welding in the temporary welding shop. Design of experiment is done by selecting voltage $(\mathrm{V})$, welding consumable feed speed $(\mathrm{mm})$, and travel speed $(\mathrm{cm} / \mathrm{mm})$ as continuously controllable factors with low and high values as defined in Table 1 . These controllable factor's effects are studied on four responses such as weld bead ( $\mathrm{mm})$, bead reinforcement height $(\mathrm{mm})$, penetration depth $(\mathrm{mm})$ and deposition efficiency(\%). Each response is assigned with minimum and maximum values which are obtained from design condition for ASME B31.3 process piping for non-sour and non-lethal service and are mentioned in Table 2. The response's results are studied and analyzed on mean values basis and target to maximize the mean of the depth of penetration, deposition efficiency. The 
quantitative relation between responses and factors in term of Response Surface Methodology (RSM) can be expressed as below:

$$
Y=f(\text { Voltage, wire feed, travel speed })
$$

whereas $Y$ is the responses which are to be optimized and $V, F \& S$ are the controllable factors. The system behavior will be obtained through quadric model or by higher order polynomial model which is developed by the least square method by considering the interaction of factors to maximize or minimize the response variables, below is the quadratic model for author case:

$$
\begin{gathered}
Y=\beta 0+\beta 1(\text { Voltage })+\beta 2(\text { wire feed })+\beta 3(\text { travel speed })+\beta 11(\text { volatge } 2)+\beta 22(\text { wire feed } 2) \\
+\beta 33(\text { travel speed })+\beta 12(\text { voltage* wire feed })+\beta 13(\text { volatge*travel speed })+\beta 23(\text { wire feed travel speed })
\end{gathered}
$$

In Equation (1), the author designate betas as coefficient of linear, quadratic and interaction of input $V$, $F$, and $T$. The $\beta 0$ is the intercept term whereas $\beta 1, \beta 2, \beta 3$ and $\beta 11, \beta 22, \beta 33$ are the linear terms and interaction between variables terms respectively. Design of experiment's run and the model was established

Table 1. Defining of controllable factors to be measured

\begin{tabular}{||cccccc||}
\hline Name & Units & Type & Role & Low & High \\
\hline \hline A:Welding Voltage & $\mathrm{V}$ & Continuous & Controllable & 29.0 & 34.2 \\
B: Wire Feed Speed & $\mathrm{mm}$ & Continuous & Controllable & 3.9 & 9.7 \\
C:Welding Speed & $\mathrm{mm} / \mathrm{min}$ & Continuous & Controllable & 50.0 & 70.0 \\
\hline \hline
\end{tabular}

Table 2. Defining of responses to be varied

\begin{tabular}{||cccccccc||}
\hline Name & Units & Analyze & Goal & Impact & Sensitivity & Low & High \\
\hline \hline Depth of Penetration & $\mathrm{mm}$ & Mean & Maximize & 3.0 & Medium & 4.5 & 6.0 \\
Deposition Efficiency & $\%$ & Mean & Maximize & 3.0 & Medium & 55.6 & 93.4 \\
Bead Width & $\mathrm{mm}$ & Mean & Maximize & 3.0 & Medium & 6.5 & 8.0 \\
Bead Reinforcement & $\mathrm{mm}$ & Mean & Maximize & 3.0 & Medium & 0.7 & 3.0 \\
\hline
\end{tabular}

by Box-Behnken design which was developed by George E. P. Box. Box-Behnken is one of the experimental strategies for RSM where each factor is placed at equally distributed values on at least three levels and it fit on the quadratic model the design is equidistant from the design center which is placed in last. Total 30 runs are designed with 3 center points per block. The Design of experiments (DOE) parameters are tabulated in Table $3[8]$.

Table 3. Design of experiments for a gas metal arc welding process

\begin{tabular}{||lccccccc||}
\hline $\begin{array}{l}\text { Type of } \\
\text { Factors }\end{array}$ & Design Type & $\begin{array}{c}\text { Center points } \\
\text { Per Block }\end{array}$ & $\begin{array}{c}\text { CenterPoint } \\
\text { Placement }\end{array}$ & $\begin{array}{c}\text { Design is } \\
\text { Randomized }\end{array}$ & $\begin{array}{c}\text { Number of } \\
\text { Replicates }\end{array}$ & Total Runs & Total Blocks \\
\hline \hline Process & Box-Behnken design & 3 & Last & Yes & 1 & 30 & 2 \\
\hline
\end{tabular}

To fit the results of the experiment, the Quadratic model of factors interaction is used and below table is the statistical data collected from large data points of the mobile welding shop, 30 runs are collected and responses of each run are measured from actual testing in mechanical laboratory refer to Table 4 for detail. The welding was conducted on API 5L Gr. 70 (fine grain Normalized 19mm thickness) material with single bevel angle. Bevel angle was checked with Dye penetrant testing for any possible defects like crack etc. GMAW machine of model MAXI 505 was used for welding. Whereas reinforcement was measured by using Cambridge type welding gauge.

The welding joint design is given below in Figure 2; (joint design and bead width and reinforcement height etc [2].)

The larger pool of field data for GMAW process is reported in Raw Data file by authors [9]. 


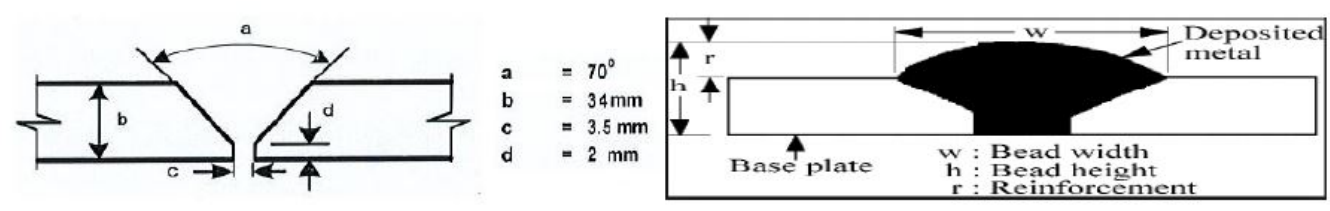

Figure 2. Joint Design Detail used for GMAW Welding

Table 4. GMAW data for optimization process

\begin{tabular}{|c|c|c|c|c|c|c|c|}
\hline \multirow[t]{2}{*}{ Sr. No. } & \multicolumn{3}{|c|}{ Factors } & \multicolumn{4}{|c|}{ Responses } \\
\hline & $\overline{F 1}$ & F2 & F3 & R1 & R2 & R3 & R4 \\
\hline & Voltage (V) & Wire Feed & Travel Speed & $\begin{array}{l}\text { Depth of } \\
\text { Penetration } \\
(\mathrm{mm})\end{array}$ & $\begin{array}{c}\text { Deposition } \\
\text { Efficiency } \\
(\%)\end{array}$ & $\begin{array}{l}\text { Bead Width } \\
\quad(\mathrm{mm})\end{array}$ & $\begin{array}{c}\text { Weld } \\
\text { Reinforcement } \\
(\mathrm{mm})\end{array}$ \\
\hline 1 & 29 & 6.8 & 50.0 & 5 & 55.6 & 6.50 & 0.7 \\
\hline 2 & 34.2 & 6.8 & 70.0 & 6 & 91.6 & 6.50 & 2.0 \\
\hline 3 & 29 & 9.7 & 60.0 & 5 & 90 & 6.00 & 2.0 \\
\hline 4 & 31.6 & 3.9 & 50.0 & 5.5 & 77 & 8.50 & 3.0 \\
\hline 5 & 29 & 3.9 & 70.0 & 5 & 77 & 8.00 & 2.5 \\
\hline 6 & 31.6 & 3.9 & 50.0 & 4.5 & 56 & 6.50 & 0.8 \\
\hline 7 & 34.2 & 6.8 & 70.0 & 5.5 & 92.5 & 6.50 & 2.2 \\
\hline 8 & 34.2 & 9.7 & 60.0 & 6 & 92 & 6.00 & 2.2 \\
\hline 9 & 31.6 & 9.7 & 50.0 & 6.5 & 80 & 8.00 & 3.0 \\
\hline 10 & 31.6 & 9.7 & 60.0 & 5.3 & 92.3 & 6.50 & 2.0 \\
\hline 11 & 34.2 & 3.9 & 70.0 & 5.2 & 92.1 & 6.40 & 2.0 \\
\hline 12 & 29 & 6.8 & 50.0 & 5.3 & 92.3 & 6.50 & 2.0 \\
\hline 13 & 31.6 & 6.8 & 60.0 & 5 & 92.2 & 6.50 & 2.0 \\
\hline 14 & 34.2 & 9.7 & 70.0 & 5.3 & 88.7 & 7.0 & 3.0 \\
\hline 15 & 31.6 & 3.9 & 50.0 & 5.6 & 90.2 & 7.7 & 2.9 \\
\hline 16 & 31.6 & 6.8 & 60.0 & 5.3 & 90.7 & 6.3 & 2.5 \\
\hline 17 & 34.2 & 3.9 & 70.0 & 5.3 & 88.6 & 7.8 & 3.1 \\
\hline 18 & 29 & 6.8 & 50.0 & 6.0 & 94.7 & 7.3 & 2.7 \\
\hline 19 & 31.6 & 9.7 & 60.0 & 6.1 & 94.0 & 9.4 & 2.9 \\
\hline 20 & 34.2 & 9.7 & 70.0 & 6.1 & 94.6 & 6.1 & 2.5 \\
\hline 21 & 31.6 & 3.9 & 50.0 & 5.7 & 93.0 & 8.5 & 2.9 \\
\hline 22 & 31.6 & 6.8 & 60.0 & 5.2 & 88.3 & 6.3 & 2.9 \\
\hline 23 & 34.2 & 6.8 & 70.0 & 5.2 & 87.7 & 7.0 & 3.0 \\
\hline 24 & 29 & 9.7 & 50.0 & 5.2 & 89.1 & 6.3 & 2.5 \\
\hline 25 & 31.6 & 3.9 & 60.0 & 5.4 & 89.4 & 7.7 & 2.9 \\
\hline 26 & 34.2 & 6.8 & 70.0 & 5.8 & 93.3 & 7.3 & 2.7 \\
\hline 27 & 29 & 3.9 & 50.0 & 6.3 & 96.5 & 9.6 & 3.4 \\
\hline 28 & 31.6 & 6.8 & 60.0 & 5.9 & 95.2 & 6.6 & 2.6 \\
\hline 29 & 34.2 & 9.7 & 70.0 & 6.3 & 98.9 & 10.5 & 2.6 \\
\hline 30 & 31.6 & 3.9 & 50.0 & 5.6 & 91.7 & 6.6 & 2.8 \\
\hline
\end{tabular}

\section{Analyze the Experiments Results [8]}

The variance's analysis is performed by Analysis of variance (ANOVA) was conducted to investigate the controllable factor's influences on measured responses then the standardized Pareto chart is drawn for each response with respect to significant factors. The analysis of variance is calculated for each of response and is explained in detail, refer below to Table 5 for depth of penetration, Table 6 for deposition efficiency, Table 7 for bead width and Table 8 for weld reinforcement height: Table 5 is the variance analysis known as ANOVA where statistical significance is calculated by associating the experimental error with a mean square. The mean square value was obtained by computing the variability in Sq-Root (DOP) into a distinct run for each of the effects. In author occasion, no interactions of factors have P-values less than 0.05 which confirmed that all interactions are meaningfully diverse from " 0 " at the $95.0 \%$ confidence level. 
Table 5. Variance's analysis for SQRT of Depth of Penetration

\begin{tabular}{||cccccc||}
\hline Source & Sum of Squares & Dof & Mean Square & F-Ratio & P-Value \\
\hline \hline A:Welding Voltage & 0.0330452 & 1 & 0.0330452 & 1.73 & 0.2799 \\
B:Wire Feed Speed & 0.0384051 & 1 & 0.0384051 & 2.01 & 0.2512 \\
C:Welding Speed & 0.0113129 & 1 & 0.0113129 & 0.59 & 0.4975 \\
AA & 0.00000229293 & 1 & 0.00000229293 & 0.00 & 0.9919 \\
AB & 0.00715199 & 1 & 0.00715199 & 0.37 & 0.5838 \\
AC & 0.00036437 & 1 & 0.00036437 & 0.02 & 0.8989 \\
BB & 0.0000164209 & 1 & 0.0000164209 & 0.00 & 0.9784 \\
BC & 0.000137468 & 1 & 0.000137468 & 0.01 & 0.9377 \\
CC & 0.0020654 & 1 & 0.0020654 & 0.11 & 0.7639 \\
Total error & 0.0572911 & 3 & 0.019097 & & \\
Total (corr.) & 0.150612 & 12 & & & \\
\hline
\end{tabular}

The R-Squared calculation shows that the model as fitted illuminates $59.7 \%$ of the variability in Sq-Root (Depth of Penetration). The adjusted R-squared calculation is 39.55\%, The standard error of the calculation indicates as 0.0871038 . The mean absolute error of 0.0590065 is the average value of the residuals. Based on

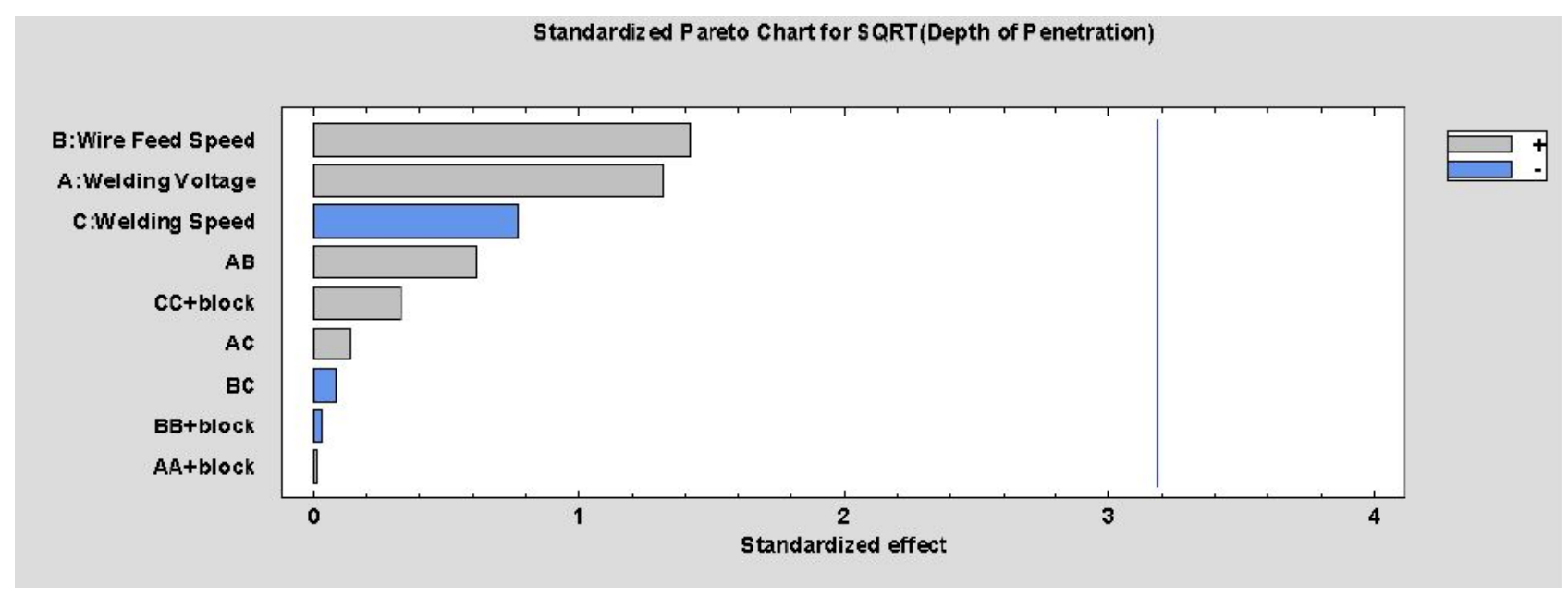

Figure 3. Pareto Chart for SQRT of the depth of penetration

analysis of variance through ANOVA, the significant controllable factors are identified and then plotted against the standardized effect. Figure 3 shows that Wire Feed, welding voltage and their quadratic effect have a major effect on depth of penetration as compared to other factors. P-value for all interactions are greater than $5.0 \%$. Table 6 is the variance analysis known as ANOVA where statistical significance is calculated by associating the

Table 6. Variance's analysis for SQRT of Deposition Efficiency

\begin{tabular}{||cccccc||}
\hline Source & Sum of Squares & Dof & Mean Square & F-Ratio & P-Value \\
\hline \hline A:Welding Voltage & 1.16387 & 1 & 1.16387 & 2.45 & 0.2158 \\
B:Wire Feed Speed & 1.12495 & 1 & 1.12495 & 2.36 & 0.2217 \\
C:Welding Speed & 0.272031 & 1 & 0.272031 & 0.57 & 0.5045 \\
AA & 0.00838265 & 1 & 0.00838265 & 0.02 & 0.9028 \\
AB & 0.128551 & 1 & 0.128551 & 0.27 & 0.6391 \\
AC & 1.20742 & 1 & 1.20742 & 2.54 & 0.2094 \\
BB & 0.205795 & 1 & 0.205795 & 0.43 & 0.5577 \\
BC & 0.95518 & 1 & 0.95518 & 2.01 & 0.2515 \\
CC & 0.821663 & 1 & 0.821663 & 1.73 & 0.2802 \\
Total error & 1.42735 & 3 & 0.475783 & & \\
Total (corr.) & 7.44221 & 12 & & & \\
\hline \hline
\end{tabular}


experimental error with a mean square. The mean square value was obtained by computing the variability in Sq-Root (DOP) into a distinct run for each of the effects. In author occasion, No interactions of factors have P-values less than 0.05 which confirmed that all interactions are meaningfully diverse from âĂIJ0âĂİ at the 95.0\% confidence level.

The R-Squared calculation shows that the model as fitted illuminates $80.82 \%$ of the variability in Sq-Root (Depth of Penetration). The adjusted R-squared calculation is $23.22 \%$, The standard error of the calculation shows 0.689771 for the standard deviation of the residuals. The mean absolute error of 0.271215 is the average value of the residuals. Based on analysis of variance through ANOVA, the significant controllable

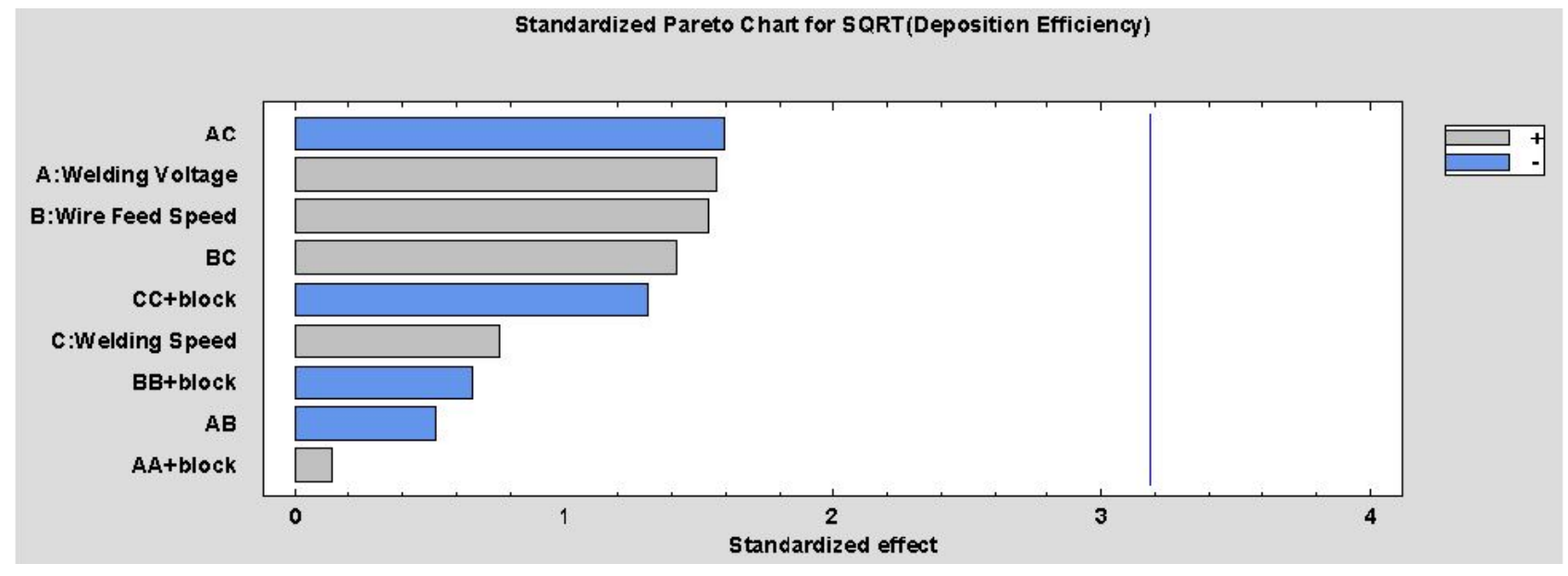

Figure 4. Pareto Chart for SQRT (Deposition Efficiency)

factors are identified and then plotted against the standardized effect. Figure 4 shows that quadric effect of Welding voltage and wire feed and wire feed and welding speed have a major effect on deposition efficiency as compared to other factors. P-value for all interactions is greater than $5.0 \%$. The R-Squared calculation

Table 7. Variance's analysis for SQRT of Bead Width

\begin{tabular}{||cccccc||}
\hline Source & Sum of Squares & DOF & Mean Square & F-Ratio & P-Value \\
\hline \hline A:Welding Voltage & 0.0111456 & 1 & 0.0111456 & 0.42 & 0.5650 \\
B:Wire Feed Speed & 0.037308 & 1 & 0.037308 & 1.39 & 0.3232 \\
C:Welding Speed & 0.0519844 & 1 & 0.0519844 & 1.94 & 0.2581 \\
AA & 0.0122513 & 1 & 0.0122513 & 0.46 & 0.5475 \\
AB & 0.0222912 & 1 & 0.0222912 & 0.83 & 0.4291 \\
AC & 0.0 & 1 & 0.0 & 0.00 & 1.0000 \\
BB & 0.0177043 & 1 & 0.0177043 & 0.66 & 0.4760 \\
BC & 0.00189437 & 1 & 0.00189437 & 0.07 & 0.8076 \\
CC & 0.0122513 & 1 & 0.0122513 & 0.46 & 0.5475 \\
Total error & 0.0804485 & 3 & 0.0268162 & & \\
Total (corr.) & 0.271784 & 12 & & & \\
\hline
\end{tabular}

shows that the model as fitted illuminates $70.3999 \%$ of the variability in Sq-Root (Depth of Penetration). The adjusted R-squared calculation is $0.0 \%$, which is appropriate for analyzing matrix with dissimilar number of independent variables. The estimated standard error for standard deviation of the residuals to be 0.163756 . The mean absolute error of 0.0639226is the average value of the residuals.

Based on analysis of variance through ANOVA, the significant controllable factors are identified and then plotted against the standardized effect. Figure 5 shows that Welding speed and wire feed have a major effect on bead width as compared to other factors. There is no figure for serial autocorrelation in the residuals at significance level of $5.0 \%$ because P-value for interactions is greater than $5.0 \%$. The R-Squared calculation shows that the model as fitted illuminates $44.9561 \%$ of the variability in Sq-Root (Depth of Penetration). The adjusted R-squared (the appropriate one) calculation is $0.0 \%$, .The estimated standard error of the residuals to be 0.392887 . The mean absolute error of 0.156633 is the average value of the residuals. Based on analysis 


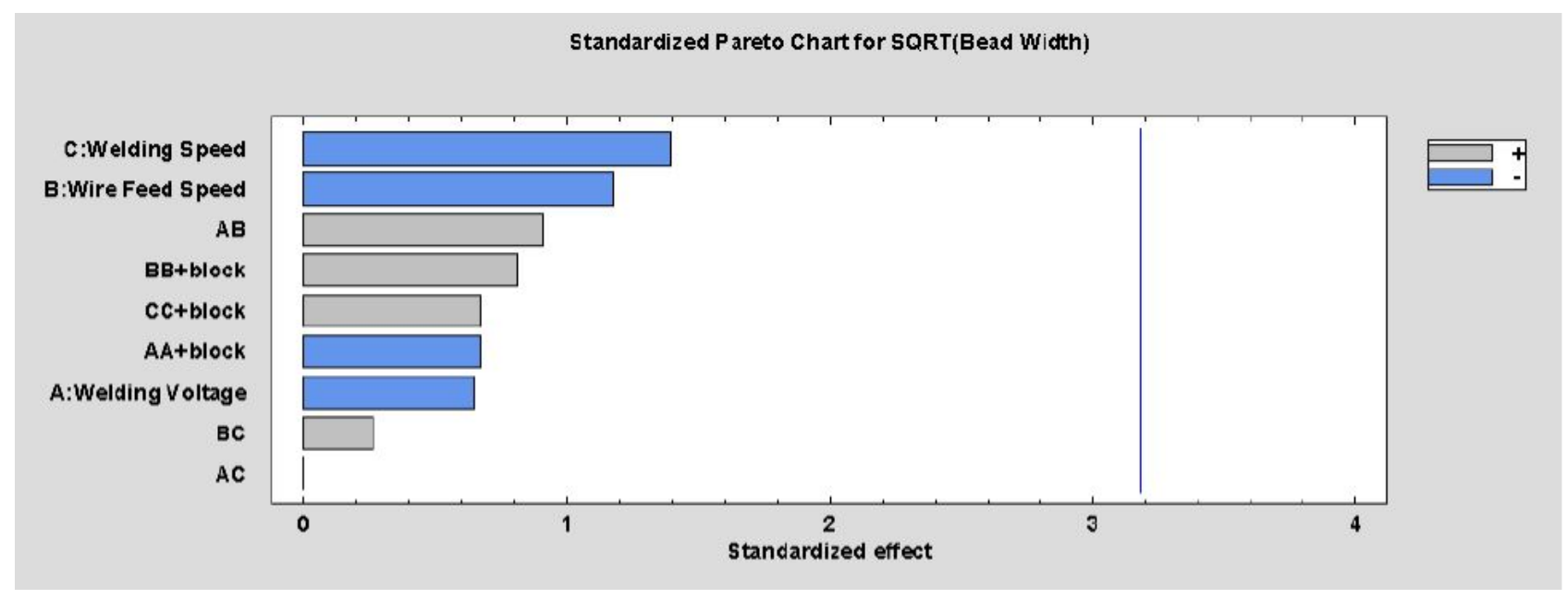

Figure 5. Pareto Chart for SQRT of Bead width

Table 8. Variance's analysis for SQRT of Weld Reinforcement

\begin{tabular}{||cccccc||}
\hline Source & Sum of Squares & DOF & Mean Square & F-Ratio & P-Value \\
\hline \hline A:Welding Voltage & 0.0376313 & 1 & 0.0376313 & 0.24 & 0.6554 \\
B:Wire Feed Speed & 0.0222486 & 1 & 0.0222486 & 0.14 & 0.7295 \\
C:Welding Speed & 0.0523154 & 1 & 0.0523154 & 0.34 & 0.6013 \\
AA & 0.00538944 & 1 & 0.00538944 & 0.03 & 0.8637 \\
AB & 0.0139183 & 1 & 0.0139183 & 0.09 & 0.7836 \\
AC & 0.104516 & 1 & 0.104516 & 0.68 & 0.4709 \\
BB & 0.0264368 & 1 & 0.0264368 & 0.17 & 0.7068 \\
BC & 0.0675445 & 1 & 0.0675445 & 0.44 & 0.5556 \\
CC & 0.0141117 & 1 & 0.0141117 & 0.09 & 0.7821 \\
Total error & 0.46308 & 3 & 0.15436 & & \\
Total (corr.) & 0.841292 & 12 & & & \\
\hline
\end{tabular}

of variance through ANOVA, the significant controllable factors are identified and then plotted against the standardized effect. Figure 6 shows that Quadric effects of Welding voltage and welding speed have a major effect on bead width as compared to other factors.

\section{Optimization through Desirability-Function [8]}

Optimum factors setting values are obtained through the value of optimized desirability which is obtained by considering the combined results of all five responses for specific predicted values. For optimization of response values, we have selected confident level of $95 \%$. Whereas predicted values of each response is a mean value of upper $95 \%$ and lower $95 \%$ limit. Observed desirability is calculated from observed values of all response's values for each run of an experiment by using Derringer's model. Whereas predicted desirability is obtained from predicted values of all response base on lower and upper 95\% limits of the confidence level.

\section{Desirability Function [9-11]}

The desirability function and loss function are used optimized approaches and we have used desirability function methodology because it is more applicability and flexibility as compared to loss function moreover it is a most suitable function to solve multi-objective optimization problems. As per definition is given by Harrington [9], "converts every response's value into scale-free value is known as desirability". The desirability function was also studied and shared by Derringer and Suich's [10] which is used for nominal-the best (NTB), 


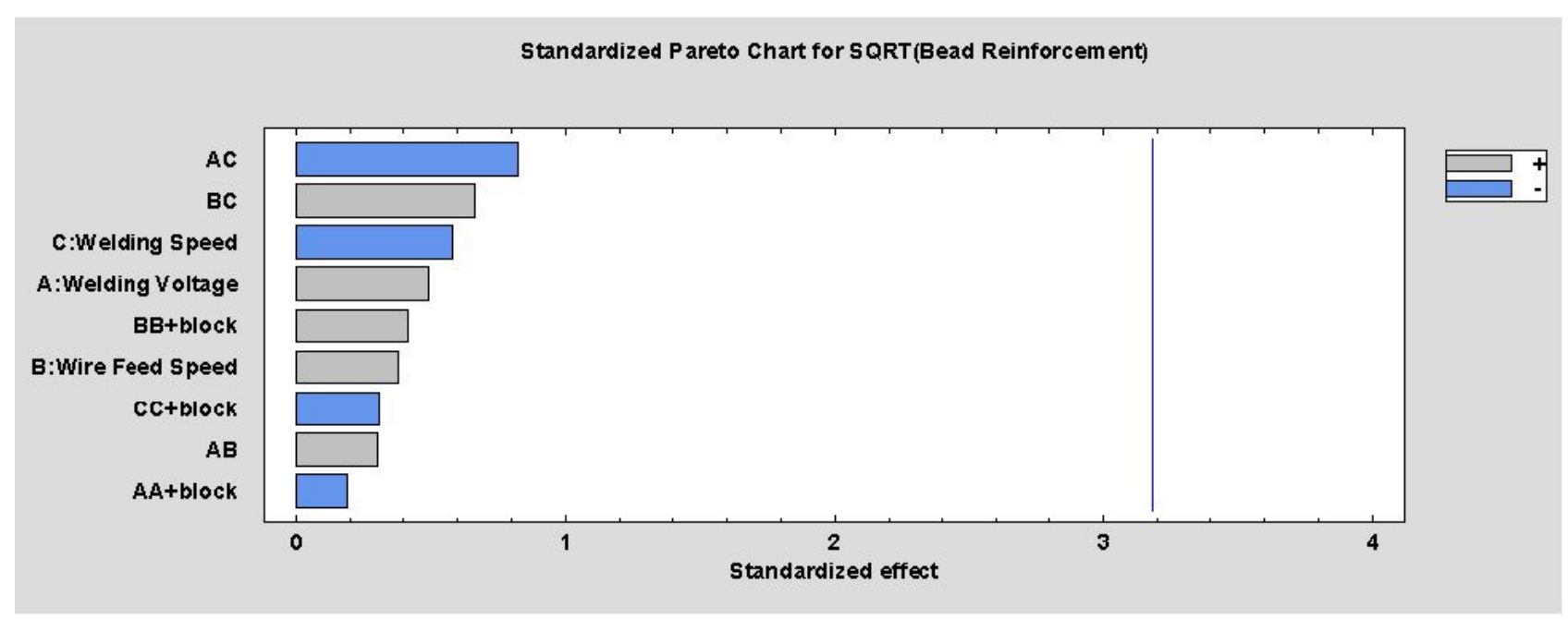

Figure 6. Pareto Chart for SQRT of Reinforcement height

larger-the-best (LTB), smaller-the-best (STB) type of measurable responses. In our DOE, we have selected the larger the best which is defined as:

$$
d(i)= \begin{cases}0, & y l \leq L S L_{i} \\ \frac{\hat{y}-U S L_{i}}{L S L_{i}-U S L_{i}}, & L S L \leq \hat{y} \leq U S L i i \\ 1, & y l=U S L_{i}\end{cases}
$$

Where $d(i)$ is the desirability function, yet is the response, $U S L_{i}$ is the upper $95 \%$ limit and $L S L_{i}$ is the lower $95 \%$ limits.

Table 9 shows all calculation where responses are optimized by getting their prediction by taking the mean of lower $95.0 \%$ limits and upper $95.0 \%$ limit. Base on Derringer and Suich's desirability function as defined above, the desirability is calculated for depth of penetration, deposition efficiency, bead width, and bead reinforcement are $52.8 \%, 85.3 \%, 81.3 \%$ and $93.9 \%$ respectively [8].

The overall desirability or optimized desirability is equaled $77 \%$, which is obtained by considering the desirability of all responses then taking each value to the power equal to its impact, taking the product of both results, and the resultant product raises to a power equal to 1 divided by impact summation. Optimized

Table 9. Optimum Response Values

\begin{tabular}{||cccccc||}
\hline Response & Optimized & Prediction & Lower 95.0\% Limit & Upper 95.0\% Limit & Desirability \\
\hline \hline Depth of Penetration & yes & 5.2921 & 3.47671 & 7.48744 & 0.528068 \\
Deposition Efficiency & yes & 87.8636 & 51.8112 & 133.382 & 0.853534 \\
Bead Width & yes & 7.72064 & 5.11714 & 10.8577 & 0.813758 \\
Bead Reinforcement & yes & 2.8614 & 0.204658 & 8.58927 & 0.939741 \\
\hline
\end{tabular}

Desirability $=\mathbf{7 7} \%$. Optimums setting of factors are obtained based on optimized desirability vs optimized responses values and are given in Table 10 and the graphical representation is mentioned in Figure 7 The

Table 10. Factor settings at optimum

\begin{tabular}{||cc||}
\hline Factor & Setting \\
\hline \hline Welding Voltage & 33.2783 \\
Wire Feed Speed & 3.9 \\
Welding Speed & 60.0 \\
\hline
\end{tabular}

graphical presentation of optimal result shows in Figure 7 and Figure 8 . Where within the model with respect 


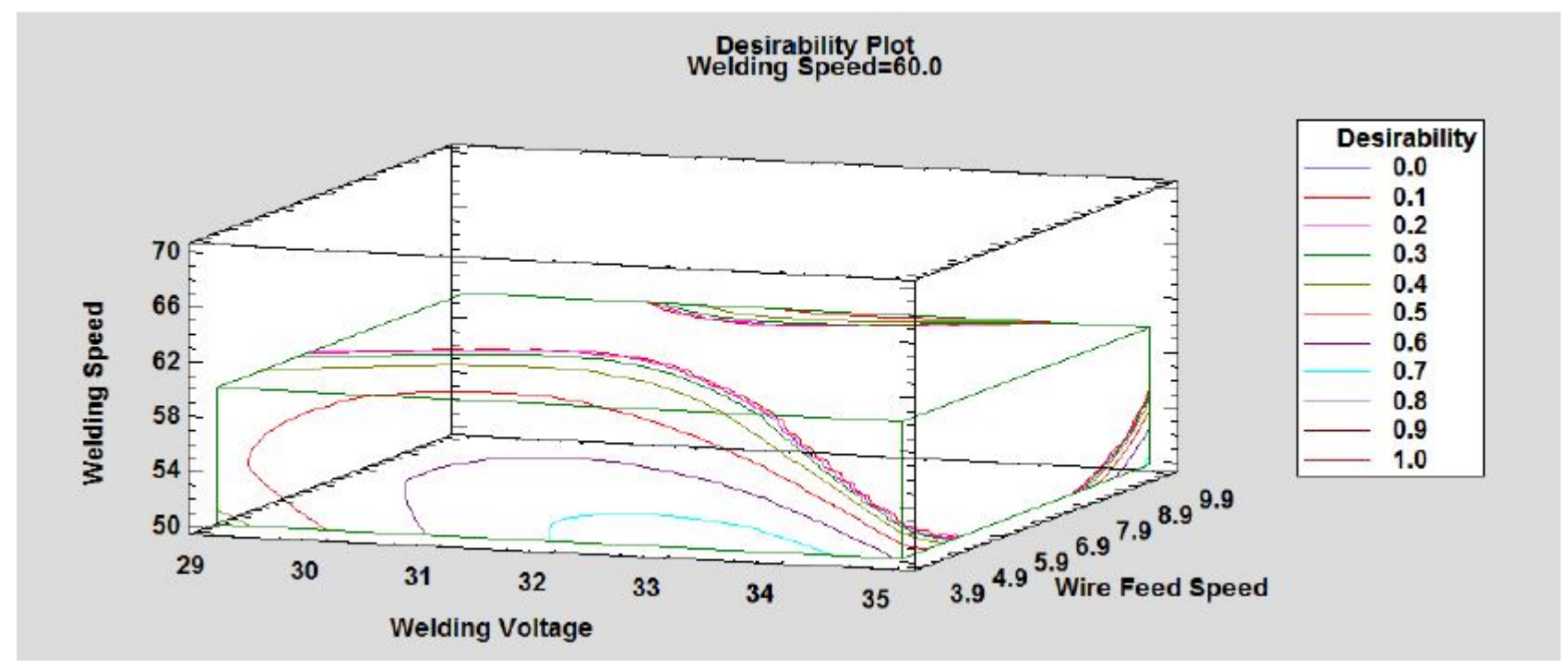

Figure 7. Optimum factors setting at the optimum desirability

to controllable welding variables desirability zone are marked in different color, blue color zone depicted the undesirable zone with value of 0 and red zone depict the ideal condition with $100 \%$ desirability. Base on welding condition and variables range, optimal desirability zone is $77 \%$ for the given values of welding parameters.

\section{Validation, Discussion, and Conclusion}

After getting the optimal values for factors next stage was to validate these values. In order to do this final weld, the run was conducted by using these optimal values obtained during these analyses. Welding was performed under same circumstances using the same material. Final results were obtained and found very close to the optimal responses as mentioned in Table 11. This study discloses the application of RSM

Table 11. Comparison between optimized and actual results

\begin{tabular}{||ccc||}
\hline Optimized Factors & Optimized Responses & Actual Results (Responses) \\
\hline \hline Welding Voltage $=33.2 \mathrm{~V}$ & Depth of Penetration $=5.2921 \mathrm{~mm}$ & Depth of Penetration $=5.3 \mathrm{~mm}$ \\
Wire Feed Speed $=3 . \mathrm{mm}$ & Deposition Efficiency $=87.8 \%$ & Deposition Efficiency $=90 \%$ \\
Welding Speed $=60 \mathrm{~cm} / \mathrm{mm}$ & Bead Width $=7.72 \mathrm{~mm}$ & Bead Width $=6.52 \mathrm{~mm}$ \\
& Reinforcement $=2.86 \mathrm{~mm}$ & Reinforcement $=2.2 \mathrm{~mm}$ \\
\hline
\end{tabular}

Box-Behnken design and desirability analyses for gas metal arc welding to optimize the welding output. By using these methodologies optimal values for selected controllable factors and responses are found and then optimal responses values are validated by performing an actual run of the weld and then comparing the actual results with the calculated optimal values. Hence conclusion can be made that for given certain material and similar welding circumstances these analyses can be used for the best quality weld.

\section{Acknowledgements}

The completion of this research work could not be possible without support and assistance of so many people whose names may not all be enumerated, however, we would like to express our thanks to particular Mr. Ali Raza lone - QC manager of Saudi Arkad, Mr. Azhar - Ph.D. student of King Fahd University of Petroleum and Minerals (KFUPM), Mr. Saravanan - JGC Department Manager of Construction and the most Mr. ITO Kenji - Senior Manager JGC for their endless support during my research work. The research work's Abstract of same methodology on Flux-cored arc welding (FCAW) was also presented in 15th Annual Congress on Materials Research \& Technology [13].

Author Contributions: All authors contributed equally to the writing of this paper. All authors read and approved the final manuscript. 


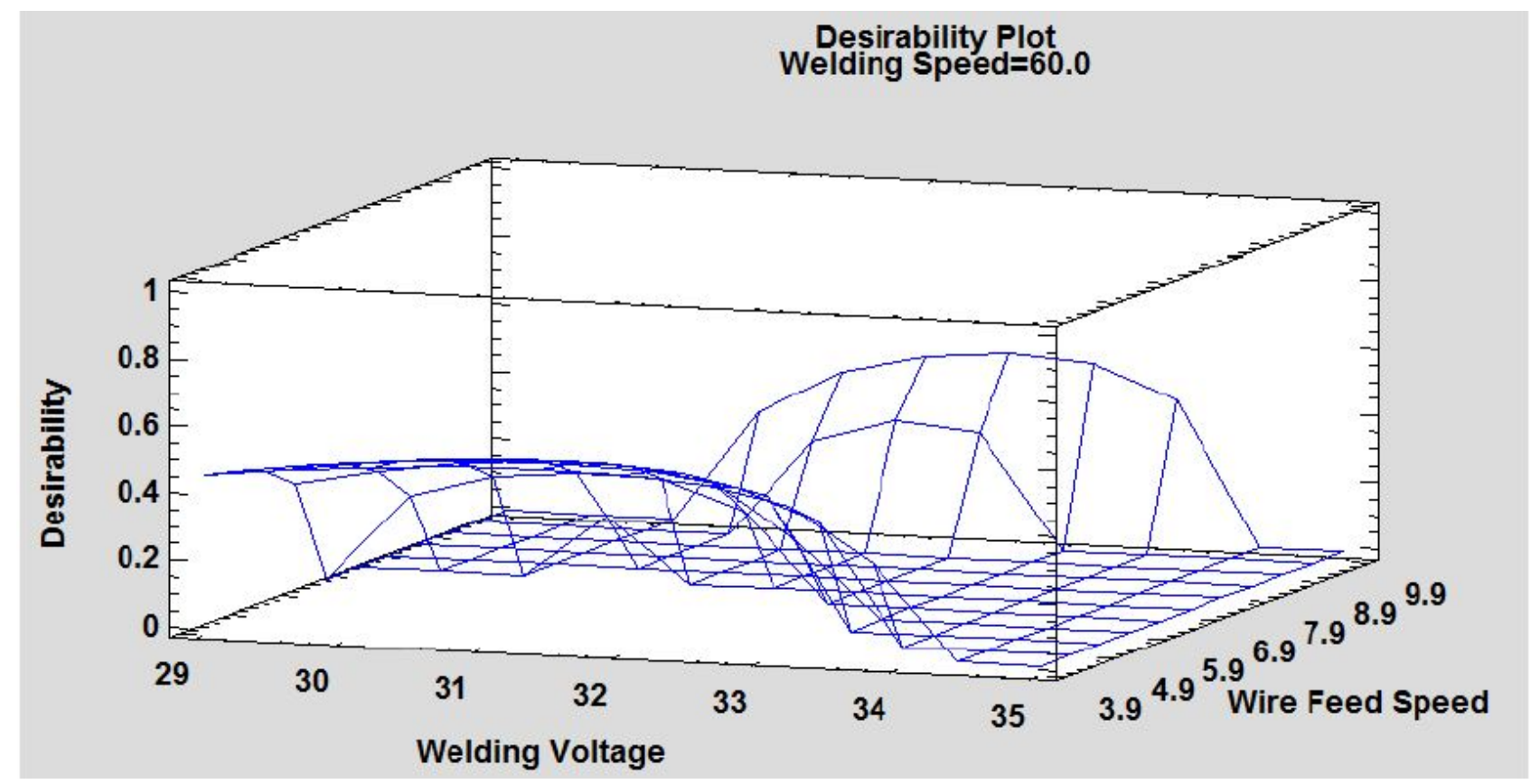

Figure 8. Optimum factors setting at the optimum desirability

Conflicts of Interest: "The authors declare no conflict of interest."

\section{References}

[1] ASMIH Committee. (1993). ASM Handbook, Volume 06-Welding, Brazing, and Soldering. ASM International, 160-164.

[2] Kou, S. (2003). Welding metallurgy. Hoboken, New Jersey: John Wiley \& Sons

[3] Moghaddam, M. A., Golmezergi, R., \& Kolahan, F. (2016). Multi-variable measurements and optimization of GMAW parameters for API-X42 steel alloy using a hybrid BPNN-PSO approach. Measurement, 92, 279-287.

[4] Bataineh, O., Al-Shoubaki, A., \& Barqawi, O. (2012). Optimising Process Conditions in MIG Welding of Aluminum Alloys Through Factorial Design Experiments. Latest Trends in Environmental and Manufacturing Engineering, 21-26.

[5] Ibrahim, I. A., Mohamat, S. A., Amir, A., \& Ghalib, A. (2012). The Effect of Gas Metal Arc Welding (GMAW) processes on different welding parameters. Procedia Engineering, 41, 1502-1506.

[6] Kumar, P., \& Roy, B. K. (2013). Parameters Optimization for Gas Metal Arc Welding of Austenitic Stainless Steel (AISI 304) \& Low Carbon Steel using Taguchi's Technique. International Journal of Engineering and Management Research (IJEMR), 3(4), 18-22.

[7] Patel, C. N., \& Chaudhary, S. A. N. D. I. P. (2013). Parametric optimization of weld strength of metal inert gas welding and tungsten inert gas welding by using analysis of variance and grey relational analysis. International Journal of Research in Modern Engineering E Emerging Technology, 1(3), 48-56.

[8] Statgraphics Centurion XVII - http:/ / www.statgraphics.com/centurion-xvii.

[9] Nazir, K. (2018). Field Data for GMAW Welding variables and responses for process optimization Studies. Research gate, DOI:10.13140/RG.2.2.29164.00644.

[10] Harrington, E. C. (1965). The desirability function. Industrial quality control, 21(10), 494-498.

[11] Derringer, G., \& Suich, R. (1980). Simultaneous optimization of several response variables. Journal of quality technology, 12(4), 214-219.

[12] Bashiri, M., Shiri, M., \& Bakhtiarifar, M. H. (2015). A robust desirability-based approach to optimizing multiple correlated responses. International Journal of Industrial Engineering \& Production Research, 26(2), 119-128.

[13] Nazir, K., Sheikh, A. k., \& Ahmad, M. A. (2018). Optimization Of flux cored arc welding (fcaw) by cube composite design and desirability function. Journal of Material Sciences \& Engineering, DOI:10.4172/2169-0022-C1-092. 